\title{
KindS OF DETERMinisM IN SCIENCE
}

\author{
ALEXANDER MAAR \\ Universidade Estadual de Londrina - UEL, BRAZIL \\ alexander.maar@web.de
}

\begin{abstract}
Determinism is a doctrine or assumption best defined in the realm of the natural sciences. In this paper I explain in detail the four senses of determinism, from the most fundamental metaphysical sense, to the most complex epistemic (predictive) sense. I take as a starting point the analysis of determinism offered by Stephen Kellert. Each of these senses is then expounded and commented with a view to explore some of the implications of each of them in theoretical physics. The most important of my tasks in this paper is to differentiate between the metaphysical and epistemic consequences of the deterministic assumption. My objective is to show that determinism as an ontological tenet is capable of withstanding criticism, even though predictive determinism is likely to be false.
\end{abstract}

Keywords: Determinism • unique evolution $\bullet$ value determinateness $\bullet$ predictability $\bullet$ quantum mechanics $\bullet$ chaos theory

\section{Introduction}

What reasons are there for holding determinism to be true of any law-governed physical system? Can determinism remain plausible despite findings in quantum mechanics suggesting that it does not hold of our actual world? Are there different senses or levels (ontological, epistemic, mathematical...) of determinism? In this article I address some of these concerns by investigating what it means for a system to evolve deterministically, and whether any such system may obtain in a quantum world.

A careful characterisation of determinism and its different senses can be found in Stephen H. Kellert's In The Wake of Chaos (1993), in particular his third chapter, "Unpeeling the layers of determinism". Such an 'unpeeling' will help us understand how the relation between ontological determinism, and predictability — the idea that there is symmetry to be found between causally explaining and predicting or retrodicting - is weakened by (fairly) recent developments in physical theory, and better philosophical analyses.

This paper starts with my exposition and interpretation of Kellert's characterisation of the various 'layers' of determinism - namely A, B, C and D. I then consider whether determinism can operate at any one or combination of these layers, and 
what the consequences are, for the doctrine of determinism, for ideas originating from chaos theory (CT) and quantum mechanics (QM). I then argue that if determinism is characterised as a metaphysical tenet, and taken to be partially obtainable, it does seem to escape refutation from criticism arising from CT and QM. I side here with John Earman's position that a retreat to metaphysics saves the doctrine of determinism from refutation $(1971,1986,2004,2007,2008)$, and I contrast his views with those of Kellert.

\section{Identifying the layers of determinism}

Determinism is sometimes used as a description of systems of a certain kind, and sometimes, more generally, in a cosmological sense. The latter requires us to consider the entire universe as a unique system. In either case, the characterisation of a system as deterministic goes through four different levels or layers, as distinguished by Kellert: A - Differential Dynamics; B - Unique Evolution; C - Value Determinateness; and $\mathbf{D}$ - Total Predictability. ${ }^{1}$

In the following sections I provide formulations for each of these theses, and comment on their philosophical implications. Although I mostly refer to Kellert's individuation of the layers, I propose a few modifications to highlight the contrast between ontological and predictive versions of determinism. I show that layer $\mathbf{A}$ is applicable to models only, layer $\mathbf{B}$ is the most fundamental and is essentially an ontological doctrine of determinism, layer $\mathbf{C}$ has a dual ontological / epistemic aspect, and layer $\mathbf{D}$ is essentially epistemic.

\subsection{Layer (A): Differential Dynamics}

A dynamical system is one whose states evolve in accordance to mathematical expressible rules (differential equations). There is a mathematical expression or rule(s) which describes, for instance, how the spatial position of a certain object evolves over time. In simple words, the state of a certain system $S$ at a time $t_{1}$, together with the relevant rules, determine the state of $S$ at $t_{2}$.

The state of the universe at time $t$ is related to the state at time $t_{0}<t$ by a mathematical expression with differential equations, because any two quantitative formulations of the 'state of the universe' are surely related by some function or other. To put teeth into this notion, it may be necessary to specify that the mathematical rule must involve simple, time invariant differential equations. (Kellert 1993, p.58)

A dynamical system is said to be deterministic if its evolution over time can be fully specified by a set of equations where no probabilities (chance) are present. As 
Kellert explains:

[...] a system is deterministic if the dynamical system that models it makes no reference to chance. [...] a dynamical system has two parts: a representation of all possible states of the system and a set of equations that describes how the state of the system changes with time. When neither of these two parts involves chance explicitly, we have a deterministic dynamical system. (Kellert 1993, p.57)

As the previous quote illustrates, for Kellert, a deterministic dynamical system is not a real physical system, but a model of a system, or as he says it, a system that models a real system - so I'll refer to it as $M_{S}$. It is a model that describes a real system's evolution according to deterministic rules. There are two requirements for $M_{S}$ to be characterised as deterministic:

1. The rules and the state descriptions must be precise, and not just approximate. Also, the state description of $S$ at a given time $t$ must include all the relevant physical information of $M_{S}$ at that time $t$.

2. If $M_{S}$ is deterministic, then there are no probabilities in which, say, the state of $S\left(t_{1}\right)$ evolves into $S a\left(t_{2}\right) 30 \%$ of the time, and into $S b\left(t_{2}\right) 70 \%$ of the time. There must be no 'branching' in the evolution of $M_{S}$ : "the rules or evolution equations must be deterministic in that they allow no [...] plurality of possibilities; they must not be stochastic equations" (Kellert 1993, p.58). ${ }^{2}$

Because $M_{S}$ is a model, and not a real system, we must not claim that the rules which apply to it are laws of nature. The key idea of layer $\mathbf{A}$ is to say that a model $M$ is deterministic if every state description of $M_{S}$ is fully deducible (in principle) by (nonstochastic) rules and the description of any prior state of $M_{S}$. In other words, we are able to deduce from a prior state of the model, a subsequent state. But we must accept the possibility that there may be a discrepancy between the model of $S$ and $S$ itself. It may well be the case that while the model seems to obey a perfectly deterministic evolution, the system being modelled could have some level of indeterminacy which we are unaware of. Determinism according to $\mathbf{A}$ is more applicable to models rather than to real systems of the world. The next layer represents best what is normally intended by determinism.

\subsection{Layer (B): Unique Evolution}

Layer B conveys the idea that determinism is a metaphysical notion applicable to real systems of the world. This notion simply says that a deterministic world, or system, is one that evolves along a unique trajectory, and if the system were, so to speak, 
be wound back to some initial state then all subsequent states would have the same history.

We can give a more precise definition. ${ }^{3}$ Let $S$ be any system which has a history over time, and let $S(t)$ be a state of the system at a given time $t$. Suppose now that there are two systems, $S$ and $S^{*}$ which have a history over time and which are governed by the same laws $L$. We may now say that $S$ is ontologically deterministic (B) if $S$ and $S^{*}$ agree at any time $t$ in their respective states (viz., $S(t)=S^{*}(t)$ ), then they agree at any other time $t^{\prime}$ (viz., $S\left(t^{\prime}\right)=S^{*}\left(t^{\prime}\right)$ ). If $S$ and $S^{*}$ disagree at any other state at $t^{\prime}$ (viz., $S\left(t^{\prime}\right) \neq S^{*}\left(t^{\prime}\right)$ ), then $S$ is indeterministic.

It may be the case that a system is only futuristically deterministic, in the sense that $t^{\prime}$ is later than $t$. Or a system may be historically deterministic, if $t^{\prime}$ is earlier than $t$. If we then take these two kinds of determinism together we can define what it is for $S$ to be overall deterministic. Thus, we can define determinism in terms of unique evolution of a system. Based on the definition given above we now can, derivatively, say what it is for a set of laws $L$ to be deterministic in terms of a system $S$ to which the laws apply. We say that $L$ is deterministic just in case the history of $S$ which they govern is (overall) deterministic. If the history of $S$ is not deterministic, but indeterministic, then $L$ is indeterministic. In sum, $L$ is deterministic only if $S$ has a unique evolutionary history. That $L$ is deterministic, and that $S$ has a unique evolution are closely tied to one another.

Lewis gives a similar account of determinism of laws deriving from uniqueness of evolution, although he expresses it in terms of possible worlds (we may take a possible world to be an all-encompassing system):

A deterministic system of laws is one such that, whenever two possible worlds both obey the laws perfectly, then either they are exactly alike throughout all the time, or else they are not exactly alike through any stretch of time. They are alike always or never. They do not diverge, matching perfectly in their initial segments but not thereafter; neither do they converge. (1986 [1979], p.37)

Determinism according to $\mathbf{B}$ is a theory about how the world evolves independently from anyone knowing that it does so, or there being any theory about that world's evolution. There has been, however, in the history of philosophy, a certain tendency to conflate the ontological version of determinism, uniqueness of evolution, with our capacity to predict the future states of a deterministic system, i.e. our ability (actual or idealised) to deduce its future states from the laws and a perfect description of the state of the world at any given time. This conflation is misleading. ${ }^{4}$ Layer B only states that the trajectory of the world (or a system) is unique - deducibility or predictability are epistemic matters which constitute a different approach to the meaning of determinism. 
John Earman makes an interesting remark here:

I take determinism to be an ontological doctrine [...] about the temporal evolution of the world. This ontological doctrine must not be confused with predictability, which is an epistemological doctrine, the failure of which need not entail a failure of determinism [as an ontological doctrine]. (2004, p.21)

The tendency to conflate the ontological doctrine with the epistemological one is, I believe, because we think of $\mathbf{B}$ as a real feature of the world (or a system), so that under ideal circumstances, deterministic systems have somewhat knowable trajectories. But as Earman points out, these are two different theses about determinism, and must not be confused. Determinism as in $\mathbf{B}$ is a real feature of the world - it says nothing about uniqueness of evolution being knowable.

\subsection{Layer (C): Value Determinateness}

The question remains whether a system in which no branching is possible, and there is only a unique path of evolution, would suffice for a definition of determinism. Well before Earman, Clark Glymour (1971) already maintained that a proper definition of determinism also needs determinateness of quantities. In simple words, such value determinateness means that all values attributed to quantities have to be exact, which is precisely where QM and determinism move in different directions.

As Glymour explains, quantum theory:

[...] does not seem to require that all its quantities have precise values at all times. The appropriate determinist attitude toward this feature of quantum mechanics is that it, too, results from incompleteness. Determinists must hold that, if the quantum theory is true, then its observables always do have precise values even though the quantum theory does not tell us what they are, and even, perhaps, though our ignorance of such values is a necessary ignorance. (1971, p.746-7)

Kellert briefly mentions in one of his footnotes the work Hans Reichenbach's The Direction of Time (1956) in which the author seems to imply that an adequate definition of determinism ought to entail B and C. As Reichenbach explains:

[Determinism] is a theory derived from observations by way of an extrapolation. Since observations lead to physical laws of great predictive power [...] if we could make more precise observations we could make predictions that would come true without exception. A set of ultimate causal connections is supposed to be hidden behind observable relationships. Determinism is thus based on an extension of observed regularities to unobserved ones; and it is assumed that the flaws of attainable predictions would vanish if we could only uncover the ultimate causal structures. (1956, p.82) 
From an idealised perspective, we could still say conditionally that if we knew all the relevant initial conditions of a system, and the ultimate physical laws involved, our prediction would reach probability 1 ; "but it is the unconditional form of causality which expresses determinism and which we must analyse" (Reichenbach 1956, p.83).

There is a logical relation between the sharpness of the values, and the prediction of the future state of the system. ${ }^{5}$ Let $D_{1}$ be the description of the state of the deterministic system $S$ at time $t_{1}$, and a description that includes numerical values of relevant properties, and $E_{1}$ be the description of $S$ at a later time $t_{2}$. Let us say that we are mostly concerned with a certain measurable parameter, say the spatial volume of some object $O$. Now, based on our actual knowledge of the numerical value of O's volume for $S\left(t_{1}\right)$ - say the volume of the object has $x$ units - and the laws, we predict with a probability $p_{1}\left(1>p_{1}>0\right)$ the volume of the object for $S\left(t_{2}\right)$, say it has a volume of $y$ units.

We may achieve a higher probability $p_{2}\left(1>p_{2}>p_{1}\right)$ by replacing $D_{1}$ with $D_{2}$, which is a more precise description of the values of all properties for the state of $S\left(t_{1}\right)$, including the volume. $D_{2}$ is more accurate because it uses, say, better measurements of the parameters involved, and/or uses an improved knowledge of the physical laws. Thanks to the more precise description $D_{2}$, we now predict with a higher probability $p_{2}$ the value of the $O$ 's volume for $S\left(t_{2}\right)$ and therefore achieve a better description $E_{2}$ of the state of $S\left(t_{2}\right)$.

Ideally, we may refine the process even further by replacing $D_{2}$ with $D_{3}, D_{3}$ with $D_{4}$ and so forth (finding higher values for $p_{3}, p_{4}, p_{5} \ldots$ ). As $D$ becomes more and more precise we are likely to move from macro to microstate descriptions of $D_{n}$. Determinism entails that for the probability $p_{n}$ to be 1 , we need to determine exact values for the volume at different times, by means of having an ultimate (complete) description $D_{n}\left(t_{1}\right)$.

The whole idea behind this is that if any of the values for the volume are indistinct, i.e. one cannot assign sharp values for O's volume at different times, the system $S$ would not be sufficiently set or fixed, or strictly speaking, deterministic. Then of course, an ultimate description for $D$ would be unattainable. Perhaps for this reason Mark Stone reformulates Reichenbach's definition establishing that for deterministic systems "the accuracy of a state description is infinitely refinable, even though any given state description will contain some error" (1989, p.125).

There is an important distinction to keep in mind. Often, when we say that the values need to be sharp, we mean that our measurements need to be accurate enough. This is an epistemic sense of value-determinateness (C), and for this reason cannot conflict with ontological determinism (B), as defined in the previous section. But C-style determinism may also have an ontological meaning: the belief that physical properties have determinate values, despite our (epistemic) failure to attribute exact numbers to (or precisely measure) all parameters. If the values are (ontologically) 
vague, we have value-indeterminism. ${ }^{6}$ This could, in principle, pose a threat to Bstyle determinism. I will come back to this issue.

\subsection{Layer (D): Total Predictability}

Layer $\mathbf{D}$ may be defined as the idea that if a system $S$ is deterministic, and if we had complete and perfect knowledge of the laws, and perfect descriptions of the various states of the system at different times, and the mental apparatus necessary to understand all the complexities involved in the evolution of $S$, and perfectly perform all the necessary calculations, then $S$ 's trajectory would be entirely predictable, or logically deducible.

The probably best-known formulation of determinism in terms of total predictability (D) is that of Laplace:

We ought to regard the present state of the world as the effect of its antecedent state and as the cause of the state that is to follow. An intelligence knowing all the forces acting in nature at a given instant, as well as the momentary positions of all things in the universe, would be able to comprehend in one formula the motions of the largest bodies as well as the lightest atoms in the world $[\ldots]$ the future and the present would be present to its eyes. (Laplace 1812, as translated in Nagel 1961, Footnote 4, p.281-282)

We can redefine Laplacian determinism using contemporary nomenclature. We can say that it is the definition of a certain variety of determinism in which the state of the system $S$ at time $t_{0}$, together with the relevant laws, uniquely determines the state of $S$ at any other time $t_{n}$ - there is a uniquely possible (relative to the laws and the initial state) sequence of states of the world, and no branching (forward or backward) is allowed. If Laplacian determinism holds, then a complete description of a system $S$ at a given instant, and the laws, completely fix the past and the future. ${ }^{7}$ This equals saying that the system is deterministic: given the way the world is 'now, the 'future' cannot (logical impossibility) be otherwise than it will be. The definition starts as an ontological thesis, but then evolves into an epistemological account.

A similar formulation of determinism had already been put forward by Leibniz about a century earlier:

$[\ldots]$ everything proceeds mathematically - that is, infallibly $-[\ldots]$ so that if someone could have sufficient insight into the inner parts of things, and in addition has remembrance and intelligence enough to consider all the circumstances and to take them into account, he would be a prophet and would see the future in the present as in a mirror. (Leibniz 1890 [c.1695], as translated in Cassirer 1956, p.12) 
Also Dalmatian scientist Roger Joseph Boscovich developed, well before Laplace, a deterministic atomic theory based on a similar characterisation of determinism:

\begin{abstract}
Any point of matter $[\ldots]$ must describe some continuous curved line, the determination of which can be reduced to the following general problem. Given a number of points of matter, for each of them, the point of space that it occupies at any given instant of time; also given the direction and velocity of the initial motion [...]; and given the law of forces expressed by some continuous curve $[\ldots]$; it is required to find the path of each of the points, that is to say, the line along which each of them moves [...] A mind which had the powers requisite to deal with such a problem $[\ldots]$ could, from a continuous arc described in an interval of time [...] derive the law of forces itself; [...] if the law of forces were known, and the position, velocity and direction of all the points at any given instant, it would be possible for a mind of this type to foresee all the necessary subsequent motions and states, and to predict all the phenomena that necessarily followed from them. (Boscovich 1966 [1763], p.141)
\end{abstract}

The problem with these three definitions is that they conflate determinism as an ontological notion (B) with prediction (D). This is a challenge for determinism, because if prediction fails, then it would appear that determinism fails. We can avoid this by keeping the two theses separate. As Earman explains, the conflation originates "from a failure to distinguish determinism - an ontological doctrine about how the world evolves - from predictability - an epistemic doctrine about what can be inferred [...] about the future (or past) state of the world from a knowledge of its present state" (2007, p.1389).

Laplace, Leibniz and Boscovich seem to believe that the truth of $\mathbf{B}$ entails the truth of D. In other words, if a system is perfectly deterministic, they say it is (in principle) deducible by a powerful ideal mind. This entailment relation does not seem obvious to me. There is no contradiction into thinking of a possible world which is ontologically deterministic, and yet allows for no deducibility. Furthermore, layer D requires a knower, and I previously said that $\mathbf{B}$ is a doctrine about the evolution of the world independent of there being any knower.

Laplacian determinism is still a useful characterisation of determinism as D-style determinism. But remember that $\mathbf{C}$ added an extra requirement to the formulation of determinism. According to $\mathbf{C}$ determinism requires that all parameters of a system need to have sharp values. So, we can now redefine the formulation of $\mathbf{D}$ in order to include $\mathbf{C}$. The predictability of a deterministic system involves: (1) all the relevant parameters must have sharp values, (2) knowledge of the applicable deterministic laws, (3) complete information of the state of the system at a given time, and (4) the ability to make the necessary calculations which yield predictions. If (1), (2), (3) and (4) obtain, then all states of the system (future and past) are logically deducible by 
a powerful (ideal) knower. Observe that layer $\mathbf{D}$ only holds if layers $\mathbf{B}$ and $\mathbf{C}$ hold. If $\mathbf{B}$ and/or $\mathbf{C}$ fail, then there can be no predictive determinism of the kind in $\mathbf{D} .^{8}$

Total predictability is, of course, an idealisation. Practical predictability is, nevertheless, possible in many cases, and quite often is a task given to scientists. ${ }^{9}$ We often possess sufficient knowledge of the laws and the initial state of some systems, in order to perform decently accurate predictions. ${ }^{10}$ But for some deterministic system (chaotic systems), our attempts to predict their future states consistently fail. This is by no means reason to infer indeterminism. Our failure to make predictions does not undermine determinism as a metaphysical tenet. One reason for our practical failure to predict is obvious; prediction is problematic because of our limited measurement devices and practical impossibility of assigning exact quantitative values to all the relevant physical parameters.

\section{Additional considerations}

All these four layers (A, B, C and D) offer distinct, and sometimes complementary, insights on the meaning of determinism. One of the most useful aspects of Kellert's exposition of determinism as dividable into different layers is precisely to show that such layers are separable. The separation is such that it is a mistake to think that if one layer holds, then the next layer holds as well, i.e., that one layer necessarily entails another - although it is reasonable to say that layer $\mathbf{D}$ presupposes layer $\mathbf{B}$.

It may be the case that we have $\mathbf{A}, \mathbf{B}$ and $\mathbf{C}$, but still do not get $\mathbf{D}$. In other words, there may be a system describable by means of deterministic differential equations (A), which follows a uniquely possible trajectory (B), and has determinate values for all relevant parameters involved (C), and of which we have a good deal of knowledge (of the laws and the state of the system at a time), but we consistently fail to predict its future states. Chaos theory offers plenty of examples of such systems.

Dynamic systems (A), on the other hand, are not real systems, so they obviously cannot have any implication on determinism as uniqueness of evolution (B), which is a real feature of the world. Similarly, by establishing unique evolution we do not imply - and this is an epistemic matter and therefore not part of $\mathbf{B}$ - that we can assign exact values for all the relevant physical parameters (C). I also said that layer C may have an ontological sense; parameters do have determinate values, although we may not know what these are. A minimal degree of value determinateness from an ontological perspective - seems to be presupposed by $\mathbf{B}$.

Earman explains this last point well: "Determinism does not presuppose sharpness of values, for we can understand determinism as a doctrine about the evolution of set or interval valued magnitudes as well as about point valued magnitudes" (Earman 1986, p.226). This requires a refinement of the definition given in $\mathbf{B}$. The unique 
evolutionary path need not be linear but within a specified volume of evolutionary space. Earman also says next that "determinism does seem to presuppose some minimal amount of determinateness; if the world were entirely a froth of potentialities $[\ldots]$ one would be at loss to say whether determinism held or failed" (Earman 1986, p.226).

Finally, I would like to once more discuss the relation between layers B and D. I said that the ontological view is separable from epistemological and semantic considerations. This is based on Montague's view that a purely ontological characterisation of determinism would not necessarily imply deducibility. Montague explains that determinism would entail that "for any instants $t_{0}$ and $t$, there are sentences $\phi\left(t_{0}\right)$ and $\phi(t)$, expressing the state of the universe at $t_{0}$ and $t$ respectively, such that $\phi t$ is deducible for $\phi t_{0}$, combined with the laws". This, in turn, would require that there be as many sentences $\phi$ as there are instants $t$ (real numbers). "But there are only denumerably many sentences, [and] there are more than denumerably many real numbers [in standard languages]." Montague then concluded that "Laplace's allegation has been shown to be quite trivially false" (1974 [1962], p.303-4).

In defence of views such as Laplace's (which I say conflates the ontological thesis of determinism with the epistemological thesis), one may say that Laplace is only using an epistemic notion (predictability) to make sense of the ontological thesis (uniqueness of evolution), and it is a highly idealised notion. So, the ontological thesis of determinism would seem to be closely tied together with predictability in principle. This would, for some, give deducibility/predictability an ontological status - I take this to be Kellert's view. My attempt is, however, to provide a characterisation of $\mathbf{B}$-style determinism that is purely ontological, and that does not make any commitment to there being deterministic theories and an all-powerful ideal mind with the computability powers to perform the deductions. But I do concede that if the deterministic laws are indeed part of the system they govern (as a real relation of the system's properties), and if there is such a powerful mind, and if there are non-standard languages capable of providing all the necessary descriptions, ${ }^{11}$ then one may well argue that under such ideal circumstances, $\mathbf{D}$ perfectly captures the evolutionary history that is required in B. Nevertheless, I am mostly concerned with practical predictability, or predictability in its ordinary, non-idealised, sense. The decision on the ontological status of deducibility is, for my purposes, not crucial.

\section{Determinism and chaotic systems}

The notion of chaos has changed over the centuries, and this perhaps explains why some people associate chaos with randomness or chance. In Classical Greek (khaos) it was defined as "abyss, that which gapes wide open, is vast and empty" (The Online 
Etymology Dictionary, n.d.). In late 14c., Old French chaos retained the sense of a 'gaping void'. Up to this point, chaos had a strong ontological sense, and was often used to define the nature of the universe before the 'Creation'. In around 1600 its meaning shifted and extended to connote 'utter confusion' (The Online Etymology Dictionary, n.d.) i.e. lack of order, a disorganised state of affairs.

Chaos theory employs a modern, mathematical use of the notion (c.1977), which contrasts sharply with its earlier meaning. In this modern sense, a chaotic system is simply one whose trajectory through state space is highly sensitive, or highly dependent on its initial conditions, in the sense that minor unobservable changes to these may yield major (observable) effects. There is no implication that the system is not organised, or that objective chance is present, although predictions of future states of the system are very difficult. There is something in the nature of a chaotic system that prevents finite knowers from improving their epistemic situation. So CT has ontological and epistemic implications; chaotic systems may be entirely deterministic, and yet unpredictable (deterministic chaos).

The origin of this concept is to be found in that of linearity. In simple terms, the laws of nature are said to be linear if the so-called 'effects' are proportional to the so-called 'causes'. So, if linearity is the case, a billiard ball hit twice as hard as usual, moves twice as quickly, for instance, a case easily represented by a linear function. It would be correct to say that such a function represents an approximation, since we are not taking into account a number of variables; the friction of the ball with the table, or the slight differences in the angle the ball gets hit, or the resistance of the air, and so on. But if the system is sufficiently stable (not highly sensitive to variations) the linear function yields a sufficiently accurate prediction.

A number of non-linear problems in classical mechanics were solved, at least until the beginning of $20^{\text {th }}$ Century, by approximation using linear functions. A good example is the case of planetary motion. In the case of two planets gravitationally attracting each other, classical physics can explain the attraction using linear functions, and this yields successful predictions. But when a third planet comes into play, classical mechanics mathematics cannot account for it. Astronomers practically dealt with the problem by using the attraction exerted by the sun on the second planet as a basis, while considering the third planet's force a perturbation they could not account for. Their predictions were not very accurate, but approximate, because of the nature of the laws governing planetary motion. Newton's 'three-body problem' represents a case where a deterministic system - the planetary motion of planets - is governed by nonlinear laws. Therefore minor changes to the motion of Venus, for instance, could result in major unpredictable alterations to the motion of other planets.

The meteorologist Edward Lorenz (1993) popularised the idea of high sensitivity to initial conditions ('the butterfly effect') using the example that predicting the weather can be very difficult, because meteorologists deal with cases of deterministic 
nonlinearity, or deterministic chaos. The mapping of a chaotic system's trajectory is very irregular, and might give the impression of randomness or chance, but in reality it is driven by deterministic forces. ${ }^{12}$ There are two conditions for deterministic chaos: we need at least three dynamical variables, and the laws must be non-linear.

The failure to yield an accurate prediction due to the complexity of a non-linear system is epistemic. In the case of a linear system, the accuracy of any prediction depends on how well a scientist knows the laws and the relevant initial conditions: the more complete the information, the more accurate the prediction. Deterministic chaos acts as an amplifier; because any minor 'cause' yields a large effect, the predictions will be less and less accurate as time unfolds. And because chaotic systems are unstable, any minor intervention may yield large unpredictable consequences, and yet this need not be the result of indeterminacy, but could be that of non-linearity combined with 'multiple variables' or 'interacting components'. The issue at hand here is not whether the instability is generated by genuine indeterminacy or deterministic chaos; the worry is about prediction (D). The meaning of chaos is somewhat independent from that of determinism and indeterminism, and only seems to conflict with one of the layers of determinism, as I shall now discuss.

Bearing in mind the fourfold division of the senses in which the doctrine of determinism gets used, we may proceed to some useful comparisons. Chaotic systems are normally linked to layers A, B, and C (to some degree), but not D. Chaotic systems are representable by dynamical systems, they are shown to have a unique historical trajectory, or evolution, to have value determinateness, but are practically unpredictable. Therefore, if CT is true, then $\mathbf{D}$ does not obtain: "even a system characterised by point values that evolve along a unique trajectory according to straightforward differential equations will be unpredictable if small differences in initial conditions lead to widely separated trajectories later on" (Kellert 1993, p.62).

Chaotic systems are by definition highly sensitive-dependent on differences to the initial conditions, and because no measurements are in effect conducted with total accuracy, from an epistemic perspective total predictability is impossible. It is important to note, however, as Earman rightly says, such high sensitivity, which causes the chaotic evolution of a system, in itself "does not contradict the continuous dependence of solutions on initial data, and, therefore, does not undermine the task of predicting with any desired level of accuracy [...] assuming that error in measuring the initial conditions can be made arbitrarily small" (Earman 2007, p.1390).

The practical impossibility of predicting chaotic behaviour (evolution) is explained by the fact that our measurements are not perfect, and no matter how small the inaccuracy in ascertaining values for the initial conditions, the forecast of the future state of the world for a chaotic system (even assuming deterministic chaos) increasingly degrades as time unfolds. Epistemic determinism (predictability) fails.

Classical physics normally represents deterministic systems well when it talks of 
problem cases in which perfectly accurate measurements, combined with layers $\mathbf{A}$, B and C, lead to total predictability. ${ }^{13}$ This is an idealisation in which we assume to have exact measurements, and powers of precise calculation (no average numbers, approximations, cutting corners, etc.), so that even slight alterations to initial condition could be accounted for (see Hunt 1987, p.132). Practical predictability of real system is never total predictability, and this is especially so in the case of chaos.

The dissociation of ontological and epistemic versions of determinism constitutes Earman's central defence of the doctrine of determinism when compared to CT: "determinism and prediction need not work in tandem; for the evolution of the system may be such that some future states are not predictable [...] although any future complement than the one fixed from eternity is impossible" (Earman 1986, p.9).

In a later paper Earman says the following:

Ontological determinism is compatible with sensitive dependence on initial conditions. When measurement procedures for ascertaining the values of state variables are not error free ${ }^{14}[\ldots]$ the link between determinism and prediction is weakened and even broken. Furthermore, a strong form of sensitive dependence on initial conditions [...] plus the compactness of phase space implies 'chaos' [... ] On a macroscopic scale a deterministic system can behave in a seemingly random and stochastic fashion. This raises the issue of whether and how critters such as us can be justified in believing that the stochastic behaviour we are observing is due to indeterminism in the form of an irreducibly stochastic element or to deterministic chaos. (Earman 2004, p.27)

Our failure to predict the future is an epistemic matter, and a separate matter from determinism understood ontologically. It is, therefore, not incoherent to argue that if we had complete knowledge of all relevant physical properties of a given system, and complete knowledge of the laws, and had all the requisite powers of computability to make the deductions, then any apparently erratic behaviour (evolution) could be explained away as a case of deterministic chaos, and not a result of inherent indeterminacies of nature.

As previously said, the failure to predict with total accuracy need not imply the failure of $\mathbf{B}$ : a system which behaves so as to manifest high dependence on even minor alterations to its initial conditions is practically unpredictable because scientists cannot foretell which unique trajectory the systems will follow. But that there is uniqueness remains a plausible theory, only D-style determinism seems implausible.

Because of the difficulty in obtaining $\mathbf{D}$ we might be well justified in trying to define determinism, fundamentally, according to the layers A, B and C. Of these, uniqueness of evolution (B) is the most important layer for Earman. Our inability to ascertain which uniquely possible path a chaotic system will follow over time has no effect whatsoever on the (metaphysical) doctrine that the future and past states of 
a deterministic system (even chaotic ones) are uniquely fixed according to the laws. It should now be easy to see that $\mathbf{B}$ is more fundamental; if $\mathbf{B}$ holds of some system, then we may expect to model a deterministic system's rules in terms of differential equations (A), hope to attribute determinate values to relevant parameters (C), and perhaps talk of prediction under an idealised model such as Laplace's. If B holds it would offer support for A, C and D. Because Earman's defence of determinism is metaphysical - and not the result of empirical observation - its fulfilment depends solely on "the structure of the world, independently of what we could [...] know of it [...] ontological determinism does have epistemological implications [...] but let us not confuse the implications of the doctrine with the doctrine itself" (Earman 1986, p.7-8).

Kellert mentions, in opposition to Earman, the work of Ilya Prigogine. Such a reference is a useful step towards our understanding of chaotic systems as allegedly posing a threat not only to $\mathbf{D}$, but also to $\mathbf{B}$. During an inaugural lecture titled "The Arrow of Time," Prigogine (1999) explains why he suspects the deterministic worldview, according to layer $\mathbf{B}$, not to hold of the actual world. This has to do with socalled 'bifurcations' in history - there are known phenomena represented by highly unstable systems. In such systems the role of probability is evident: "in front of a bifurcation, you have [... ] many branches. The system 'chooses' one branch; if you repeat the experiment it may choose another branch. The choice of a branch is associated to probability" (Prigogine, 1999). So, if objective chance or probability is part of the equation, the future (and past) is not fixed, as it is governed by probabilistic rules.

As Prigogine then argues, this may give some useful insights into domains other than the physical sciences:

[... ] in biology [... ] Jean-Louis Deneubourg has made very nice experiments [...]. Imagine an ant nest, a source of food, and two bridges. You see that after some time all ants are on one bridge. Should you repeat the experiments, they may be on the other bridge. The mechanism is again an autocatalytic mechanism because each ant encourages the other ants to be on the same bridge. This is a very simple example of a bifurcation in biology. Also, human history is full of bifurcations. When we went from the Palaeolithic Age to the Neolithic Age due to the fact that humans could explore the resources of vegetation and of metallurgy, we may consider this as a bifurcation; even as a bifurcation with many branches, because the Chinese Neolithic is different from the Middle East Neolithic or the Latin American Neolithic [... ] In fact, one can probably say that every time we find a new resource [coal, electricity...] [...] the world is reorganised, and we have a bifurcation. (Prigogine, 1999)

In this brief excerpt, Prigogine has provided a useful example of indeterminism 
in the history of society. This is not to say that the example is true, but it accurately describes indeterminism in history by rejecting uniqueness of evolution. I suggest the following illustration for Prigogine's claim (Figure 1).

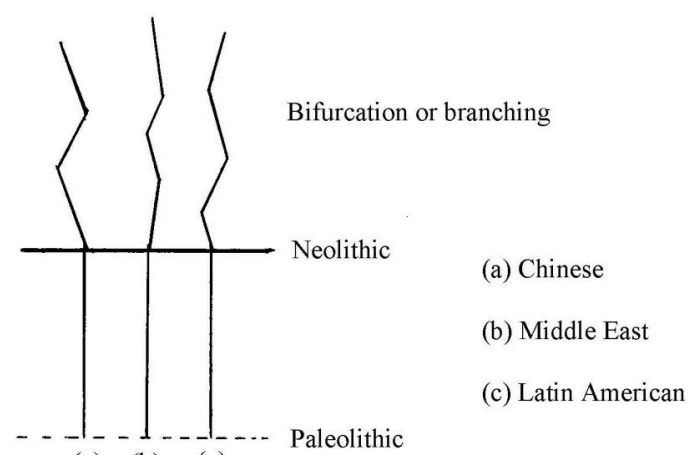

(a) (b) (c)

Figure 1: Bifurcations in the history of societies.

This diagram is, of course, an idealisation; we must think of (a), (b) and (c) being exactly alike up to the Neolithic, and equally obeying the applicable laws. The example illustrates what is meant by a bifurcation. Until reaching the Neolithic, the evolution or trajectory of Chinese (a), Middle Eastern (b) and Latin American (c) societies were the same - they followed the same unique trajectory. Then, with the agricultural revolution and the possibility of exploring new resources, (a), (b) and (c) evolved differently; we have a bifurcation or branching, there is not a uniquely possible path of evolution anymore. This fits the definition of indeterminism. As previously seen, if three systems share the same conditions at any given time $t$ (which $\mathrm{a}, \mathrm{b}$ and $\mathrm{c}$ do until reaching the Neolithic), and are governed by the very same laws, for determinism to hold all three systems need to have identical states at any other time $t_{n}$; if they are dissimilar it's a case of indeterminism.

It is hard to assess the value of such insights into the non-physical domains of knowledge. In the case of biology, the ants' behaviour (in a certain respect only) could appear random at macro-level, but it could be non-random (deterministic) at micro-level. ${ }^{15}$ In the case of history the situation is even more complicated, and the 'branching' identified by Prigogine as starting from the finding of new resources need not occur at random. It is also hard to see whether all the relevant variables involved can be properly quantified and 'packaged' into equations (probabilistic or not).

For Prigogine the deterministic worldview is false because strictly deterministic trajectories are not observable. ${ }^{16}$ This is a weak epistemic objection. B-style determinism can hold ontologically though it is not observable. Since our measurements 
always suffer from some degree of inaccuracy, it is never the case that we can specify with complete precision the state of $S$ at a future time $t$ based on our knowledge of the state of $S$ at a previous time $t^{\prime}\left(t^{\prime}<t\right)$. Sufficiently unstable systems quickly render approximations obsolete.

Prigogine is in fact challenging the distinction between layers $\mathbf{B}$ and $\mathbf{D}$ (in its ordinary sense), claiming that these cannot be separate; so if $\mathbf{D}$ is false, then this also has to be the case for $\mathbf{B}$. This strategy is mistaken if determinism is defined only as an ontological doctrine (B). Prigogine is not willing to concede the separation of the problem of determinism from that of prediction (as defended by Earman). In the case of highly unstable systems (chaotic) "everything takes place, physically speaking, as if the knowledge of [...] [the initial] data would not determine the [system's evolution]" (Hadamard 1923, p.38). As Kellert is quick to point out, if systems behave as if the present does not determine the future, determinism seems to be challenged. ${ }^{17}$

Earman's response to this is rather simple. If we look into the equations used in classical mechanics, and we are capable of seeing how real systems would satisfy the condition of uniqueness postulated by layer (B), we have a plausible metaphysical definition of determinism, which is not challenged by the fact that total prediction (D) is not practically achievable - prediction and determinism belong to different categories. ${ }^{18}$ As a consequence, chaotic systems alone only challenge epistemological accounts of determinism.

\section{Value determinateness, dynamic evolution and quantum theory}

One of the most salient differences between classical and quantum mechanics concerns dynamic, state-dependent properties of objects (position, momentum, energy, etc.). In QM it is just not possible to assign sharp values to the properties of a quantum object, and the state of an object is characterised as a wavefunction, i.e. a mathematical description of the quantum state of a quantum system. The wavefunction is a complex-valued probability amplitude, and the probabilities for the measurements made on the system can be derived from it. As the theory goes, wavefunctions can be added together and multiplied by scalars, and form a Hilbert space.

The temporal evolution of the wavefunction is governed by a linear wave equation, the Schrödinger equation (which is deterministic). But the state-evolution of a typical quantum object is random and not governed by it. In fact, it can only be ascertained post factum. How so? The wavefunction defines all possible observable states of a quantum system, and before any measurement is done, there is no reason to attribute any higher degree of reality to any one possible state - they are all equally 'permitted' as observational outcomes by the wavefunction. But upon measurement 
we get just one out of the many possibilities QM offers. ${ }^{19}$ This is known as the measurement problem: "an experimental situation in which a physical entity undergoes an indeterministic and irreversible change, called the collapse of the wavefunction" (de Bianchi, 2015). The collapse is required to connect QM with reality.

Here lies the locus of $\mathbf{D}$-indeterminism in QM: "even in the presence of exact knowledge of the current situation, it is impossible to predict its future exactly [i.e. the state of the quantum object at the completion of the measurement process], regardless of how much work and care one invests in such a prediction" (Styer, 1999). The best one can hope for is to attach probabilities to the possible final states. This is attained by means of a rule of correspondence (the Born rule). So, if orthodox (Copenhagen) interpretation of QM is correct, we certainly have $\mathbf{D}$-indeterminism.

Let us now turn our attention to layer $\mathbf{C}$. The fact that QM cannot ascribe precise values to all dynamical properties of quantum objects led some (Schrödinger and Einstein) to believe the theory was somehow incomplete, whereas others (Bohr and Heisenberg) insisted that value indeterminateness (C-indeterminism) was indeed a property of such objects. ${ }^{20}$ Layer $\mathbf{C}$ presupposes that each parameter of a system has precise, sharp values for all observables, for any state of a system at any time. Kellert appeals to QM to show that either determinism as requiring such sharpness of values is false, or we have to revise our definition for determinism once more, this time eliminating layer $\mathbf{C}$ as a requirement. Can determinism still survive in a world where there is ontic value indeterminateness? Earman argues that it can be so; determinism is not undermined by the failure of value determinateness. Even if the world is as QM represents it to be: "a universe where systems with somewhat 'spread out' properties evolve along uniquely determined paths retains coherence" (Kellert 1993, p.68-9).

This is a point of contention in the sense that the minimal amount of value determinateness necessary for a system to be characterised as deterministic seems to be arbitrary. I can imagine that some philosophers would maintain that even a small amount of value indeterminateness would account for a system not being, strictly speaking, deterministic. How much value determinateness is required by uniqueness of evolution is an important issue for which I can offer no contribution. But even if C-determinism fails, one cannot easily conclude that ontological (B) determinism is false. One distinction to keep in mind is that $\mathbf{C}$-indeterminism can be simply an epistemic doctrine - we do not know what more precise values can be obtained though they are there to be got. ${ }^{21}$ But it could also have an ontological meaning, as one may argue that some properties are themselves vague in their values. If vagueness is not epistemic, if some object is indeed vague (exhibits vagueness as a property), ${ }^{22}$ then ontic vagueness must be part of any true description of it.

In "Can There Be Vague Objects?" (1978) Gareth Evans famously argued against the existence of vague objects with a proof modelled after Kripke's refutation of contingent identity. The argument, a reductio ad absurdum, can be reconstructed as fol- 
lows: If ontic vagueness is true, then some statement of the form ' $a=b$ ' must be vague. We know that $a=a$ is true. It follows that $a$ has the property of being identical to $a$. Since $a=b$, then $b$ must have the property of being identical to $a$. Therefore we must conclude that $a=b$ is true.

The conclusion is obviously false (it contradicts Leibniz's Law). Evans argued that it is incoherent to assert that there are objects about which it is a matter of fact that they have vague or fuzzy boundaries. The argument gave rise to a heated philosophical debate. ${ }^{23}$ Technical objections have been raised on the basis that Evans is relying on inferences that are challenged by deviant logicians. ${ }^{24}$ Evans's proof can be challenged on other grounds as well. Jonathan Lowe (1994) offered a well-known refutation of Evans's analysis, showing where the formal error presumably lies. But more importantly, he offered a counterexample, a quantum-theoretical situation which makes the case for ontic vague identity:

Suppose that in an ionisation chamber a free electron $a$ is captured by a certain atom to form a negative ion which, a short time later, reverts to a neutral state by releasing an electron $b \ldots$ according to [QM] there may simply be no objective fact of the matter as to whether or not $a$ is identical with $b \ldots$ in such an interaction electron $a$ and other electrons [enter] a 'superposed' state in which the number of electrons present is determinate but the identity of any one of them with $a$ is not, thus rendering likewise indeterminate the identity of $a$ with the released electron $b$. (1994, p.110)

Lowe's example is useful, because it shows how ontic vagueness, or C-indeterminism, arises in QM. Chibeni, in "Ontic Vagueness in Microphysics" (2004) offers other scientific examples of ontically vague quantum objects which do not involve the identity relation, ${ }^{25}$ and concludes that:

Our best contemporary theory on the structure of matter, quantum mechanics, clearly ascribes fuzzy properties to objects [and] several theoretical and experimental results in microphysics afford very strong evidence for the existence of vague objects, as they prove that any theory purporting to restore sharpness in the properties of quantum objects will meet with severe constraints. (Chibeni 2004, p.38)

Chibeni is referring here to the shortcomings of hidden variable theory (HVT). Initiated by von Neumann (1955 [1932]), this programme adds additional 'components' - theories where quantum indeterminism is replaced by deeper deterministic descriptions - to eliminate some of QM's unwanted features: indeterminism, nonlocality, and the dependence between the observer of a quantum system and the properties of the system itself. HVT's aims are to build more complete models that agree with QM's predictions while restoring $\mathbf{C}$ and $\mathbf{D}$-style determinism. The desired 
outcome is to demonstrate that randomness is just observer ignorance, and not a feature of nature. ${ }^{26}$ The HVT programme was implemented more fully by David Bohm in the early 1950s and achieved success in restoring sharp values to dynamic properties of quantum objects. ${ }^{27}$ But as Chibeni remarks:

This achievement has a price: certain other theoretical and conceptual traits of classical theories are violated by the theory [...] further theoretical and experimental research has revealed that not only Bohm's theory has to pay this price, but any other theory capable of completing the QM property assignment must pay as well [...] There is a series of algebraic proofs ['no-go' theorems] to the effect that completing the quantum states through hidden variables leads to inconsistencies. [Furthermore] in 1964 John S. Bell proved that the most objectionable trait of Bohm's theory, nonlocality, must be present in any HVT reproducing certain quantum mechanical predictions. (Chibeni 2004, p.34)

The blows HVT received from the formulation of the 'no go' theorems, and from experimental results, made the programme lose steam. But it also prompted to this day the search for alternative explanations of how to 'complete' QM. Diederik Aerts, to mention a recent example, proposed to associate the hidden-variables not to the state of the measured quantum object, but to its interaction with the measuring apparatus - the 'hidden-measurements interpretation of quantum mechanics' (Aerts \& de Bianchi, 2014). As de Bianchi optimistically explains, if the proposal can be made to work, then "the no-go theorems no longer apply, and it becomes possible not only to conceptually explain the nature of a quantum measurement, but also to derive, in a non-circular way, the Born rule" (2015). The proposal has been met with scepticism.

The rich and complex theoretical issues introduced by HVT and alternative theories are of great philosophical interest, but a technically accurate discussion of the programme, its achievements and limitations, falls beyond the scope of this paper. ${ }^{28}$ The message I wish to convey is that $\mathbf{C}$-determinism survives the quantum challenge if vagueness is epistemic, and not a property of quantum objects. The hidden variable theorist wants to show that $\mathrm{QM}$ offers an incomplete representation of the world, and that determinism (both $\mathbf{C}$ and $\mathbf{D}$ ) can be theoretically restored in microphysics - although it may not be worth the price. Furthermore, it is not clear how or whether C-indeterminism implies B-indeterminism. Earman, as we have previously seen, believes that the layer of unique evolution can be isolated from value indeterminateness, as long as the 'spread out' possibilities for measurements of quantum properties are not arbitrarily large. ${ }^{29}$ 


\section{Local determinism}

Earman's position is that if unique evolution (B) is taken away, then we are left in a world where physicists would need to appeal to strange elements such as blind chance to provide understanding of quantum phenomena. But for Kellert, layer A alone, or what he calls differential dynamics as local determinism, would suffice to avoid Earman's worries. As Kellert explains the appeal to "nonstochastic differential equations to understand physical change urges us to seek local connections between events without concern for the global property of unique evolution" (1993, p.75).

This solution basically entails that at a local level, a subsystem, it would be possible to fix the rules for a differential dynamic system so that no branching is allowed (no probabilities involved). In other words, no branching of a systems' trajectory is no different to defining determinism as (local) uniqueness of evolution. Research into chaotic systems "takes place wholly within a context of local determinism [...] chaos theorists seek to understand complex behaviour by building models that obey precisely the strictures of differential dynamics" (Kellert 1993, p.75). This fact alone seems to contrast with an important idea that permeates Kellert's and Prigogine's position; that failure to prove determinism would result in a decrease of its methodological importance.

Local determinism is not without its problems. Even if we only consider a subsystem, for which we observe the evolution of, say, the state of a particle over time, any small amount of value indeterminacy can account for huge variations as time unfolds, resulting in an idealisation of deterministic behaviour. On the other hand, it may be that stochastic behaviour is intrinsic to quantum theory; therefore even local determinism is unattainable. ${ }^{30}$ But such a supposition is not yet sufficiently warranted.

\subsection{Partial determinism}

I wish to bring attention to the fact that talking about local determinism is not an entirely original solution, it only denies that there is something like a global or universal uniqueness of evolution. Earman says wisely that determinism "needn't be an all-ornothing affair. A world may be partially deterministic, deterministic with respects to some magnitudes [...] but not with respect to others" (1986, p.13).

Earman also provides an example of such a world. We may imagine that a world is only partially deterministic because:

$[\ldots]$ it is deterministic only with respect to the magnitudes which characterise the ordinary matter of which we and our scientific instruments are composed but not with respect to the magnitudes which characterise the behaviour of a free-spirited species of particle, the freeon, say [...] The world 
might be non-deterministic but still conditionally deterministic on a subset of magnitudes: if two worlds agree for all times on the values of the conditioning magnitudes and if they agree at any instant on the values of the other magnitudes, then they agree at any other instant [... ]. (Earman 1986, p.13)

The central idea here is that, at a conceptual level, it is coherent to say that the universe could be only partially deterministic, i.e. deterministic only "with respect to partial histories defined by the values of magnitudes in some proper subset $D \subset O$ of the occurrent physical magnitudes but non-deterministic with respect to partial histories defined by the values of magnitudes in some other proper subset $N \subset O$ " (Earman 2007, p.1371). In Earman's example $D$ and $N$ are subsets of magnitudes, or partial (sub) systems, part of a larger set of magnitudes $O$ of the system $S$. Partial determinism means that $S$ may evolve in a certain way that some of its subsets evolve deterministically, while other subsets are indeterministic.

There are some difficulties to be dealt with here. One must assume that it is theoretically possible that a system evolves like this, in the sense that the indeterministic subset does not ever interfere with the deterministic subset. It is however difficult to imagine a scenario of partial determinism if $D$ and $N$ are so-called basic magnitudes; the indeterminism of the evolution of the magnitudes in $N$ must not ever interact in any possible way with the deterministic evolution of the magnitudes found in $D$, otherwise $D$ could not possibly have a unique trajectory. If such isolation of $D$ is implausible, then we could only rescue $D$ 's deterministic evolution by postulating a theory showing that any upsetting effect of $N$ on $D$ are somehow cancelled out.

I wish to contend that there is really not much difference in postulating the possibility of a nonstochastic differential equation (local determinism, as Kellert names it) and the world being only partially deterministic, or conditionally deterministic on a subset of magnitudes (as Earman names it). Research into QM might pose reasonable doubt as to the plausibility of global uniqueness of evolutionary trajectories; but determinism is still to be defined as the non-branching trajectory of a system. It is just not possible to refer to determinism without layer $\mathbf{B}$, I believe. It may be that after all things are considered, we are incapable of identifying any single system, in the actual world, which fits our definition of determinism. If nothing fulfils the definition, then there is indeterminism. So be it. But we cannot say with certainty, at this point, that such failure is not simply the result of our cognitive limitations.

\section{Final remarks}

The primary aim of this paper was to spell out in some detail the different characterisations (layers) of determinism in the physical sciences. Such definitions of determinism should be applicable, however, to any systems, from CT and QM to systems 
in biological or even human sciences. Kellert concluded that determinism survives only as an ideal model of reality (A), so that layers B, C and D do not hold of the actual world, in the light of contemporary physics. Other authors, such as Karl Popper (1956), believe that determinism is the combination of all these layers (A, B, C and D). For Clark Glymour (1971) and Mark Stone (1989), determinism means the combinations of A, B and C, doing away with D only. John Earman (1986, 2004, 2007, 2008), however, contends that only B would suffice for determinism to hold - a position I see as perfectly coherent.

Furthermore, in association with the idea of determinism we have that of causation. As it is usually believed, in a totally deterministic world, if $c$ causes $e$, then $e$ occurs out of necessity. Not so if we have only partial determinism. If partial determinism holds true, then some events will be caused as a result of necessity, while others will be caused as a result of a combination of antecedents and probabilistic laws (or objective chance). I argued that partial determinism is a promising concept, somewhat similar to Kellert's notion of local determinism, because it explains, at least in principle, how determinism may obtain in a quantum world. Finally, I hope to have demonstrated how a retreat to metaphysics remarkably improves the longevity of the doctrine of determinism, which remains to this day a topic of considerable philosophical interest.

\section{References}

Aerts, D. \& de Bianchi, M. 2014. The Extended Bloch Representation of Quantum Mechanics and the Hidden-Measurement Solution to the Measurement Problem. Annals of Physics 351: 975-1025.

Barnes, E. 2010. Ontic Vagueness: A Guide for the Perplexed. Noûs 44 (4): 601-27.

Bohm, D. 1952. A suggested interpretation of the quantum theory in terms of 'hidden variables'. Physical Review 85 (2): 166-93.

Born, M. 1927. Physical aspects of quantum mechanics. Nature 119 (2992): 354-57.

Boscovich, R. 1966 [1763]. A Theory of Natural Philosophy. Cambridge (MA): MIT Press.

Brandenburger, A. \& Yanofsky, N. 2008. A classification of hidden-variable properties. Journal of Physics A: Mathematical and Theoretical 41 (42): 425302.

Cassirer, E. 1956. Determinism and Indeterminism in Modern Physics. Trans. O. T. Benfey. New Haven: Yale University Press.

Chaos. (n.d.) In: D. Harper (ed.), The Online Etymology Dictionary. http://www.etymonline. com/index.php?term=chaos. Access: 17/04/2014.

Chibeni, S. 2004. Ontic Vagueness in Microphysics. Sorites 15: 29-41.

de Bianchi, M. 2015. Solving the (Quantum) Measurement Problem. https://medium.com/ quantum-physics/solving-the-quantum-measurement-problem-5c24c22663b0 Access: $16 / 05 / 2019$.

Earman, J. 1971. Laplacian Determinism, or is this any way to run a universe? Journal of Philosophy 68 (21): 729-44. 
Earman, J. 1986. A Primer on Determinism. Dordrecht: Reidel.

Earman, J. 2004. Determinism: What Have We Learned and What We Still Don't Know. In: J. Campbell; M. O’Rourke \& D. Shier (ed.), Freedom and Determinism, p.21-46. Cambridge (MA): MIT Press.

Earman, J. 2007. Aspects of Determinism in Modern Physics. In: J. Earman \& J. Butterfield (ed.), Handbook of the Philosophy of Science: Philosophy of Physics. Part B, p.1369-434. Amsterdam: Elsevier.

Earman, J. 2008. How Determinism Can Fail in Classical Physics and How Quantum Physics Can (Sometimes) Provide a Cure. Philosophy of Science 75 (5): 817-29.

Einstein, A.; Podolsky, B. \& Rosen, N. (1935) Can Quantum-Mechanical Description of Physical Reality Be Considered Complete? Physical Review 47 (10): 777-80.

Everett, H. 1957. 'Relative State' formulation of quantum mechanics. Reviews of Modern Physics 29 (3): 454-62.

Fine, A. 2017. The Einstein-Podolsky-Rosen Argument in Quantum Theory. In: E. N. Zalta (ed.), The Stanford Encyclopedia of Philosophy. Winter 2017 edition. https://plato.stanford. edu/archives/win2017/entries/qt-epr/. Access: 15/05/2019.

French, S. \& Krause, D. 2006. Individuality and Non-Individuality in Quantum Mechanics. In: Identity in Physics: A Historical, Philosophical and Formal Analysis, p.139-97. Oxford: Clarendon Press.

Glymour, C. 1971. Determinism, Ignorance, and Quantum Mechanics. Journal of Philosophy 68 (21): 744-51.

Hadamard, J. 1923. Lectures on Cauchy's problem in linear partial differential equations. New Haven: Yale University Press.

Heylighen, F. 2002. Deterministic Chaos. In: F. Heylighen \& V. Turchin (ed.), Principia Cybernetica Web. http://pespmc1.vub.ac.be/CHAOS.html. Access: 22/07/2014.

Keefe, R. \& Smith, P. 1997. Introduction: theories of vagueness. In: Vagueness: A Reader, p.157. Cambridge (MA): MIT Press.

Kellert, S. 1993. In the Wake of Chaos: Unpredictable Order in Dynamical Systems. Chicago: Chicago University Press.

Kripke, S. 2000. Identity and Necessity. In: S. Stainton (ed.), Perspectives in the Philosophy of Language: A Concise Anthology, p.93-122. Peterborough (Canada): Broadview Press.

Laplace, S. 1812. Théorie Analytique des Probabilités. Paris: Courcier.

Leibniz, G. 1890 [c.1695]. Von dem Verhängnisse. In: C. J. Gerhardt (ed.), Die Philosophischen Schriften von Gottfried Wilhelm Leibniz. Volume VII, p.117-23. Berlin: Weidmann.

Lewis, D. K. 1986 [1979]. Counterfactual Dependence and Time's Arrow. In: Philosophical Papers. Volume II, p.32-52. Oxford: Oxford University Press.

Lorenz, E. N. 1993. The Butterfly Effect. In: The Essence of Chaos, p.179-82. Seattle: University of Washington Press.

Lowe, E. J. 1994. Vague Identity and Quantum Indeterminacy. Analysis 54 (2): 110-4.

Lucas, J. R. 1970. General Arguments for and against Determinism. Oxford: Clarendon Press.

Montague, R. 1974 [1962]. Deterministic Theories. In: R. Thomason (ed.), Formal Philosophy: Selected Papers of Richard Montague, p.303-59. New Haven: Yale University Press.

Nagel, E. 1961. The structure of Science. New York: Harcourt/Brace/World.

Parsons, T. \& Woodruff, P. 1995. Worldly indeterminacy of identity. Proceedings of the Aristotelian Society 95: 171-91. 
Parsons, T. 2000. Indeterminate Identity: Metaphysics and Semantics. Oxford: Oxford University Press.

Popper, K. 1956. The Open Universe. Totowa: Rowman/Littlefield.

Prigogine, I. 1999. The arrow of time. Inaugural lecture of the workshop The Chaotic Universe. http://www.icra.it/publications/books/prigogine/motivation.htm Access: 01/04/2014.

Prigogine, I. \& Stengers, I. 1984. Order out of Chaos: Man's New Dialogue with Nature. Toronto: Bantam.

Reichenbach, H. 1956. Determinism versus Indeterminism in Classical Physics. In: The Direction of Time, p.83-95. Berkeley: University of California Press.

Smith, P. 1998. Explaining Chaos. Cambridge (UK): Cambridge University Press.

Stochastic. 2009. In: The American Heritage Dictionary of the English Language. $4^{\text {th }}$ Edition. Boston: Houghton Mifflin. https://ahdictionary.com/word/search.html?q=stochastic\& submit. $x=51 \&$ submit. $y=16$. Access: 01/04/2014.

Stone, M. 1989. Chaos, Prediction and Laplacean Determinism. American Philosophical Quarterly 26 (2): 123-31.

Styer, D. 1999. Introduction to the Strange World of Quantum Mechanics. http://www. oberlin.edu/physics/dstyer/StrangeQM/intro.html. Access: 12/05/2019.

von Neumann, J. 1955 [1932]. Mathematical Foundations of Quantum Mechanics. New Jersey: Princeton University Press.

Williamson, T. 1994. Vagueness. London: Routledge.

\section{Notas}

${ }^{1}$ Kellert attributes this fourfold division to Mark Stone (1989).

2'stochastic' - To be defined as any equation containing reference to chance or probability; or equations containing one or more random variable(s). Interestingly, the word comes from Greek stokhazesthai - 'to guess at' (The American Heritage Dictionary of the English Language, 2009).

${ }^{3}$ The following definition is adapted from Earman (1986, p.12-4) and Montague (1974, p.319-36). I believe this to be a strictly ontological, non-epistemic, view on determinism, different from Kellert's characterisation of B-style determinism (1993, p.59).

${ }^{4}$ This is based on my interpretation of Montague (1974, p.303-5).

${ }^{5} \mathrm{Cf}$. Reichenbach (1956, p.84).

${ }^{6}$ There is a sense in which only the science of fundamental particles could be called deterministic as in C-style determinism, because all other sciences deal with the average of processes occurring at micro-scales.

${ }^{7}$ This needs to be expressed as a matter of the deducibility of a statement of the total state of the universe at a time $t+n$ (say) from a statement of its total state at $t$ in conjunction with a complete statement of the laws governing the system. $C f$. Earman (1971).

${ }^{8}$ Kellert says that also layer A must hold, i.e. complete knowledge of a system requires that it be quantified properly, and that the laws are formalised as differential equations.

${ }^{9}$ Scientific determinism is somehow similar to Laplace's position but does not require infinite precision. It basically says that the deterministic theory the scientist uses to predict should say what degree of precision (value determinateness) we need for our knowledge of 
the initial conditions, even before any calculations have been performed. Cf. Popper (1956, p.36).

${ }^{10}$ Earman provides an example: "there is an interesting connection between determinism and practical predictability for laws of motion that admit an initial value problem that is wellposed in the sense that, in some appropriate topology, the solutions depend continuously on the initial data" (2007, p.1389).

${ }^{11}$ Richard Montague claims that it would be preferable not to appeal to non-standard languages (1974, Footnote 1, p.304).

${ }^{12} \mathrm{Cf}$. Heylighen (2002).

${ }^{13}$ There are, however, some problem-cases in classical mechanics which are understood as conflicting with determinism. Cf. Earman (2008, p.817-29).

${ }^{14}$ As is the case of any actual measurement.

${ }^{15}$ Prigogine's example raises the question of the levels the laws operate. If we have a higherlevel law which is probabilistic in nature, it does not preclude that events occurring at the micro-level of reality are not behaving deterministically. Or, we could have some level of indeterminism in a micro-scale of events, while the macro-scale exhibits determinism. The notion of partial determinism brings some clarity to this issue, as we shall see.

${ }^{16}$ See exposition in Kellert (1993, p.64). One might well argue that this claim is verificationist.

${ }^{17} \mathrm{Cf}$. Kellert (1993, p.65). Universal determinism is challenged, at least. What seems to be challenged is whether there are any actual systems which fit the definition. Maybe our actual world is not deterministic after all. Still, this does not go against the possibility of defining determinism in ontological terms. On the contrary, we need the ontological definition to make sense of the notion of indeterminism.

${ }^{18}$ Again, this only relates to one reading of $\mathbf{D}$, i.e., predictability in its ordinary sense. It does not apply to $\mathbf{D}$ as an idealisation.

${ }^{19}$ Since there is no greater degree of reality to any possible state, it is worth asking, where did all the other possible states go upon collapse of the wavefunction? One of the most interesting answers, although not to everyone's taste, is the 'many worlds' interpretation formulated by Hugh Everett. It basically says that while we think there is just one outcome of a measurement, all of them occur, in other worlds which we cannot observe or access. $C f$. Everett (1957).

${ }^{20}$ Two famous thought experiments in favour of the incompleteness thesis were put forward in the 1930s: a thought experiment by Einstein, Podolsky and Rosen (1935), the EPR paradox, and Schrödinger's cat paradox. For a brief and clear explanation of exactly how these paradoxes aid the incompleteness argument, see Fine (2017). See also Chimeni (2004).

${ }^{21}$ Vagueness could be just a consequence of our deficiency in describing the world correctly, i.e. vagueness resides in our linguistic representations, not the world itself (a view defended by Frege, Russell and Dummett).

${ }^{22}$ Ontic vagueness can be defined in different ways. For the purposes of this paper, I take it to be similar to $\mathbf{C}$-indeterminism. For a definitional account of ontic vagueness, see Barnes (2010).

${ }^{23} C f$. Burgess (1989), Parsons \& Woodruff (1995), and Keefe \& Smith (1997, p.49ff).

${ }^{24} C f$. Parsons (2000). 
${ }^{25}$ This is important, as French and Krause (2006) have convincingly argued that identity does not apply to quantum particles.

${ }^{26} C f$. Brandenburger \& Yanofsky (2008). See also Williamson (1994) for a comprehensive explanation of why standard logic does not apply to vague language and how vagueness is a kind of ignorance.

${ }^{27} \mathrm{Cf}$. Bohm (1952).

${ }^{28}$ For a complete and accurate discussion of the various properties that can be asked of a hidden-variable model, and of which properties of empirically equivalent hidden-variable models are possible and which are not, see Brandenburger \& Yanofsky (2008).

${ }^{29}$ See the relevant discussion in Kellert (1993, p.68-71).

${ }^{30}$ This need not mean that in an indeterministic world events cannot be explained; only that predictability has to be replaced by new forms of explaining probabilistic phenomena. Chaos theory does provide understanding of indeterministic events. Cf. Kellert (1993, p.77-118). 